were attacks of faintness. Subsequently the suffering was localised in the course of the rignt sciatic nerve. Io was subject to remissions with severe nocturnal exacerbations ; there were also shooting pains at the epigastrium. The case was of alarming intensity, but recovered. In a lady aged forty-eight pain was referred to the right hip and to the right arm; it extended from the right shoulders to the fingers, and all movement caused pain.

There could be litble doubt that in these cases there was a form of neuritis. I met with other analogous instances in which there had been no history of influenza : one case in a child in whom there was severe pain in the calves, dropped feet, absolute loss of motor power, and, in fact, all the signs of neuritis of the alcoholic form. Any causation by alcohol was in this case quite out of the question; no doubt it was due to some infectious cause, and resembled the cases of peripheral neuritis, due to no traceable contagion, recorded by Dejérine and others.

In this summary of my personal experiences I have dealt with no cases of the earliest manifestations of influenza; all were in patients whose attack had passed away and who were not confined to their homes. The periods between the attack of influenza, and the manifestations of symptoms of pain varied from a few weeks to twenty months.

The evidence appears to me to confirm the view of Dr. Althans that the materies morbi of influenza resembles the syphilitic virus in its tendency to attack many parts of the nervous system after the attack is over, but surpasses the syphilitic toxine in virulence and in rapidity of action. Dr. Althaus ${ }^{2}$ has adopted the deductive method in his reasoning. Starting from certain probabilities, he has worked out the problems of the effects of the materies morbi if it should specially attack certain areas of the central nervous system. He concludes that the different forms of influenza are due to irritant poisoning of the bulb and the nerve nuclei contained in it. Adopting a converse method, that of logical induction, and taking my arguments alone from personal experience, I have arrived at a similar conclusion to Dr. Althaus in so far as the proposition is concerned--that the virus of influenza especially affects the nervous system. Leaving the question of the acute and early manifestations, however, which I agree with Dr. Althaus in considering to be due to involvements of certain areas in the medulla oblongata, it appears to me most probable that the consequent phenomena are better to be explained by inflammatory changes in certain peripheral parts of the nervous system. In regard to the visceral neuralgice, the hepatalgia, the gastralgia, and cardialgia, there are signs of localieation and, in some instances, of local tenderness that point to a local cause. In some such it seems probable that the sympathetic fibres and ganglia are alone affected. In other cases, as in those in which there seems to be temporary arrest of the heart's action, retching, vomiting, and various disturbances of digestion, it is most probable that the vagu is involved in greater or less degree; but here also the effects might be due to peripheral irritation. In the sensori-motor manifestations it can scarcely be possible to avoid the conclusion that there is in existence a form of neuritis analogous to that which is caused by many other toxines. The conclusion, therefore, which I bave come to is that the various affections I have briefly described are the remote consequences of the influenza infection, and that their proximate cause is a peripheral neuritis affecting the sympathetic ganglia and nerves, the vagus, and the sensori-motor nerve trunks.

Harley-street, $W$.

\section{ON THE TREATMENT OF SPREADING ULCER OF THE CORNEA WITH HYPOPION.}

BY RICHARD WILLIAMS, M.R.C.S.,

SURGEON TO THE LIVERPOOL EYE AND EAR INFIRM.ARY, AND OPHTHALMIC SURGEON TO 1 IIE ROYAL ALBERT EUWARD IN JIRMARY, WIGAN.

FEW affections of the eye are more rapid in their progress or more destructive in their consequences than those cases of ulcer of the cornea with a collection of pus in the anterior chamber, and yet I believe there are not many affections more amenable to prompt and appropriate treatment. propose in this paper to introduce a method of dealing with these cases which, as far as my knowledge extends, has not hitherto been in general use, and which consists, first, in the local application of quinine; and secondly, in a modification of the usual incision for the evacuation of the pus contained in the anterior chamber. I am able to speak of this method of treatment after an experience of several years, during which I thiak I have had ample reason to be satisfied with its results. As is well kaown, these cases are met with among the debilitated of all ages, most frequently as the result of traumatism. Among the colliers of the Wigan district they occur with unusual frequency, and I venture to assert, therefore, that my experience of them is above the average. In their treatment I have employed a solution of quinine during a period of upwards of ten years. The following mixture is used in nearly all cases that come under my care : $R$. quinice sulph. neutr., gr. iv.; liq. atropiæ sulph., $\mathbf{3} \mathbf{i}$. M.; ft. gutt In simple ulcers without hypopion $I$ am in the habit of prescribing eserine much oftener than atropine, but $\mathrm{I}$ in. variably prescribe the latter drug where hyporion is present, because iritis is a common feature in this condition. I doubt if either eserine or atropine has any appreciable influence upon the suppurative process. On the oti.er hand, there is hardly any thing in medicine about which $\mathrm{I}$ entertain less doubt than that quinine locally applied has an immediate and powerful influence in arresting this process and in causing the absorption of the pus already cullected in the anterior chamber. I believe I have had repeated evidence of this both in my private practice and public clinique.

It is not my intention to burden this paper with a number of cases which seem to me all to tell the same tale, but $I$ cannot refrain from quoting the following case, which I think not only shows the value of quinine, but also tends to prove that atropine and eserine by themselves have no great, if any, influence upon the process of pus formation :-

D. $J$ _ aged eighteen, was admitted into the Eye and Ear Infirmary on June 24th, 1882, for capsular cataract due to an injury five years previously. I am indebted to $\mathrm{Mr}$. Shears, who was then house surgeon, for the following notes of the case:-Soon after admission the opaque capsule was removed en masse by means of the cannula forceps, and two days later the patient, whose vision before the operation only amounted to perception of light, could count fingers with ease. The following day a hypopion appeared, and atropine and belladonna fomentations were ordered every three hours. On the 28th, the hypopion still remaining, quinine was added to the drop. -29 th: "Hypopion much smaller."-July 1st : "Hypopion gone." The quinine and atropine drops were continued until the $8 \mathrm{th}$, and eserine substituted $-12 \mathrm{th}$ : The hypopion reappeared, and the quinine and atropine drop was again used.-14th: "Hypopion gone, muddy condition of anterior chamber clearing up." - 17th: Atropine alone was ordered. - 19th: Hypopion reappeared with pain and injection; atropine and quinine drop again resorted to.22nd: "Hypopion gone."- 26th : Patient discharged. $\mathrm{V}=\frac{20}{30}$ c. $+6 \mathrm{D}$. J. $1, \mathrm{c} .+12 \mathrm{D}$. It will be noticed that in

this case the hypopion made its first appearance while the patient was under atropine, and that it quickly disappeared when quinine was added to the drop. Its reappearance took place each time when the quinine was omitted, although the atropine or eserine was still continued. In other respects the treatment was not varied, the patient taking quinine internally from the first appearance of the hypopion.

I may also mention a recent case recommended to me by Dr. Bouverie MacDonald of Liscard. Mr. McW - aged nineteen, had only complained of his eye about thirty-six hours before he was sent to me. I found an abscess of the cornea and a large hypopion. The cornea generally was hazy and the aqueous turbid. The conjunctiva was slightly chemosed and discharging muco.purulent matter. In fact, the disease had been sudden in its onset and extremely rapid in its progress, and I have no doubt that a delay of twentyfour hours would have seriously compromised the safety of the eye. I ordered quinine and atropine drops, to be used every two hours, and quinine internally three times a day. No other treatment whatever except a pad and bandage was employed. Two days later there was a marked improvement, and in four or five days the hypopion had completely dis. appeared. It may be argued that these cases of en recover under atropine or eserine, hot fomentations, and antiseptic lotions, and that they are no proof of the value of quinine locally applied. It is obvious that positive proof is practi- 
cally out of the question in cases of this description, and it is only after comparison of a long series of cases treated by hoth methods that anything like an authoritative opinion can be ventured. A vivid recollection of the frequency with which these cases went from bud to worse in my earlier years leaves no doubt in my mind of the immense superiority of the quinine treatment. I am far from saying that all cases of hypopion can be made to disperse by means of quinine; but I am constantly seeing cases in which, in my jadgment, the process is quickly arrested, and which would otherwise inevitably require operative measures. Old cases where the pus has become mixed with fibrinous material will no doubt require evacuation, but $I$ believe that even then the use of the quinine drop exerts a p rwerful influence in preventing the reaccumulation of the pus. Before leaving this part of the subject, I may say that patients make no unusual complaint of smarting in using the drops. An aqueous solution of the soluble quinine is innocuous, and can be used as frequently as necessary without the least detriment to the eye. Coming, then, to those cases where the evacuation of the pus by operation is imperatively demanded, the question arises, What is the best method of performing paracentesis? There are two methods in ordinary use: First, by making a horizontal incision, parallel with the margin of the cornea, just underneath the purulent collection ; and secondly, Saemisch's incision, which consists in passing the knife into the anterior chambar at the spot where the ulcer may happen to be and dividing it through and through, as shown in Fig. 1, the

FIG. 1.

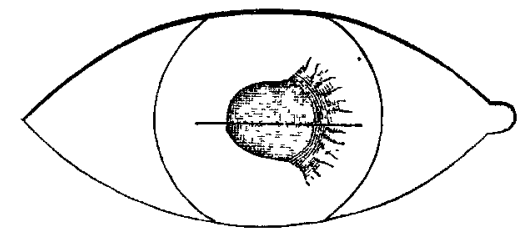

Saemisch's incision. After De Wecker.

blade entering the anterior chamber and emerging from it in healthy tissue.

To the first of these incisions I think there are serious objections. 1. The anterior chamber does not empty itself well, especially when the knife is slowly withdrawn, as is generally advised. 2. There is considerable danger of prolapse of the iris. 3. There is the maximum danger of interference with the nutrition of the cornea, because more of the nutritive channels, which are radially arranged, are involved in the incision. In fact, the only advantage this incision possesses, as far as I can see, is that it leaves no visible scar. Personally, I have entirely abandoned it for several years. Secondly, Saemisch's incision. I believe the good effect which undoubtedly follows this incision is due, not to the fact that the ulcer is cut through, but because the anterior chamber is more thoroughly emptied of the pus. On the contrary, any incision of an already attenuated and weakened part of the cornea must, it seems to me, be in itself injurious. I have not seen any reason advanced for dividing the ulcer itself as practised by Saemisch, and, unless it can be shown that this method possesses some curative influence other than is brought about by the thorough evacuation of the anterior chamber, I believe an incision in healthy tissue is decidedly to be preferred. Moreover, it is evident that if the ulcer should happen to be situated in the upper two-thirds of the cornea, the evacuation of the contents of the anterior

Fig. 2.

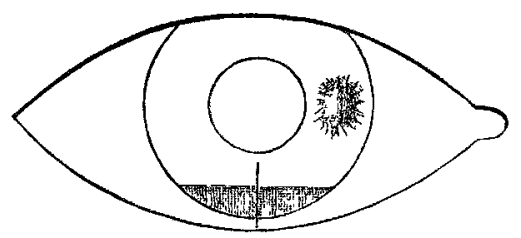

chamber must be a matter of some difficulty; and this is probably the reason why authors generally agree that the incision should be reopened daily for some time. It requires vo great knowledge of the subject to see that this reopening of the ciamber is a great drawback to the progress of the case. Is prevents healing and greatly facilitates the forma- tion of anterior synechix, not to apeak of the pain and the annoyance it gives a patient already reduced and little able to bear them.

Basing my practice on these considerations, I now al ways make the incision as shown in Fig. 2 . I introduce an ordinary Graefe's knife in to the anterior chamber underneath the puru. lent collection, and carry it directly upwards in the direction of the pupil, care being taken not to enter the pupillary area. I withdraw the kuife rather quickly with a forward motion, and the result is that the aqueous escapes with something of a rush, carrying with it the purulent mass which generally sticks between the edges of the incision, where iv can be easily picked out with a pair of forceps. Any remaining fluid pus immediately follows, and the anterior chamber is left perfectly empty. I think this incision has distinct advantages over the others to which I have referred. 1. It is made at the seat of the purulent cullection, and being in the line of pressure from behind, the evacuation is as complete as it can be. 2. Being made in the direction of the nutritive channels, it offers the minimum of interfer. ence with the nutrition of the part. 3. Prolapse of the iris is impossible, because the effect of pressure of the muscles upon the globe is to bring the edges of the incssion together, and so effectually to prevent protrusion of the iris. 4. Being made entirely in healthy structure, it heals by first intention, and I never have occasion to reopen it. If, after practising this incision, the case goes from bad to worse, it is not because the anterior chamber requires to be reopened, but because of the continued spreading of the ulcer. I have no doubt, however, as already mentioned, that the use of the quinine drop after the operation has much to do with preventing the reformation of the hypopion. In the event of the ulcerative process continuing and threatening, as it sometimes does, the entire surface of the cornea, I consider the actual cautery the most effectual weans at onr command for arresting the spreading process. In conclusion, I think the only objection likely to be advanced against this incision is that it leaves a scar; but I consider this a purely theoretical objection. The incision need never encroach upon the pupil, hence no interference with vision need be apprehended, and the scar, being linear, is practically invisible against the background of the iris.

Liverpool.

\section{SOME CASES OF RENAL COLIC.}

BY NESTOR TIRARD, M.D., F.R.C.P., SENIOR áSIETINT PHYSICILN, RING'S COLLEGE HOSPITAL; PIIYSICIAN TO TIIE EVELINA IIUSPITAL FOR SILK CHILDHEX.

IY dealing with any case of severe abdominal pain, which recurs from time to time in a paroxysmal way, it is usual to make inquiries as to the precise starting point of the pain, and the dirfetions in which it radiates, and to ascertain the occurrence of any accompanying symptoms which may throw light upon the diagnosis. The information thus gained will frequently form the foundation for further investigations, and thus the clinical features of the case may often be elucidated with ease. Thus a woman aged fifty, who was recently under my care at King's College Hospital, gave the following account of herself: Until three years ago she had had no serious illness, but at this time on several occasions she passed blood with her urine, and this hæmaturia was mostly accompanied, preceded, or followed by somewhat severe lumbar pain in the right side. After these attacks there was frequently more or less acute pain on micturition, which, however, gradually subsided. The association of hæmaturia clearly pointed to the renal origin of the colic, and, although the pain in these earlier attacks does not appear to have been as severe as usual in cases of renal colic due to calculus, there was no difficuliy in arriving at a diagnosis, since the immediate cause of her appearance at the hospital was an attack of very severe pain, during which she was repeatedly sick, and on examination the urine was found to be highly charged with blood. Mostly the passage of the first renal calculus along the ureter is attended by more pain than in later attacks, since the ureter becomes more distended each time a cal. culus passes along it, and thus offers but little opposition to the passage of a small calculus. In this 